Ann. Biol. anim. Bioch. Biophys., I973, 13 (I), 37-50.

\title{
ÉTUDE HISTOLOGIQUE DE LA CROISSANCE POSTNATALE DES MUSCLES SOLEUS ET ACCESSORIUS LATISSIMI DORSI CHEZ LE LAPIN COMMUN
}

\author{
J. NOUGUÈS \\ avec la collaboration technique de Odette Moulierac \\ Station de Physiologie animale, I. N. R. A., \\ E. N. S. A., Place Viala \\ 34060 Montpellier Cedex
}

\section{RÉSUMÉ}

La croissance postnatale des muscles accessorius latissimi dorsi et soleus a été étudiée chez des lapins mâles et femelles d'âge échelonné entre la naissance et un an.

L'étude a porté sur l'évolution des caractères suivants :

- dimensions des fibres musculaires (aire de section transversale et longueur);

- proportion de tissu conjonctif dans le tissu musculaire.

Il apparaît que :

- L'évolution en fonction du temps des aires de section des fibres musculaires a la même allure que l'évolution pondérale des muscles. Il y a une période de croissance accélérée des aires de section des fibres jusque vers 60 jours, suivie d'une période de décélération jusqu'à l'approche d'un plateau qui se situe vers 150 jours pour le soleus et vers $25^{\circ}$ jours pour l'accessorius latissimi dorsi.

Pendant la croissance des muscles étudiés, les aires moyennes de section transversale et les longueurs des fibres, ainsi que les poids frais des muscles ne sont pas significativement différents chez les lapins mâles et femelles.

La croissance en longueur des fibres musculaires est beaucoup plus précoce que la croissance en épaisseur et elle s'arrête beaucoup plus tôt. A 7o jours, pour les deux muscles étudiés, la première est pratiquement achevée.

- La proportion de tissu conjonctif, représenté par l'ensemble périmysium et endomysium, diminue dans le tissu musculaire pendant la croissance des muscles étudiés.

\section{INTRODUCTION}

La croissance du muscle dépend, principalement, de l'évolution de trois paramètres principaux caractérisant sa structure. Ces paramètres sont : le nombre de fibres, les dimensions de ces fibres - longueur et aire de sections transversales - et la quantité de tissu conjonctif. 
Dans un travail antérieur, nous avons étudié l'influence de l'évolution du nombre des fibres sur la croissance postnatale du muscle squelettique chez le Lapin (NougUìs, I972). Nous avons observé que pour certains muscles le nombre des fibres est déjà fixé à la naissance et que, pour d'autres, il y a encore une faible augmentation du nombre des fibres, qui se produit, principalement, pendant les 7 à $\mathrm{I} 7$ jours suivant la naissance. Au-delà de cet âge, on peut dire que le nombre de fibres du muscle du Lapin est définitivement fixé. Ainsi, l'augmentation de poids considérable du muscle, pendant la vie postnatale, est surtout le résultat d'une hypertrophie très rapide des fibres par une croissance en longueur et en épaisseur, accompagnée d'une accumulation de tissu conjonctif.

Ce sont ces deux paramètres structuraux que nous allons étudier, pendant la croissance postnatale du muscle chez le Lapin domestique.

Il existe de nombreuses données bibliographiques concernant la croissance en épaisseur des fibres musculaires chez diverses espèces : chez le Lapin, (MEARA, I947) chez le Porc, (Mac Meeran, I940), (Staun, I963), (Chrystall et al., I969), chez le Mouton, (Hammond et Appleton, I932), (Joubert, I956) chez le Rat, (Eliot et al., I943), (ENesco et PUdDy, I964), (ChIakulas et Pauly, I965), chez la Souris (GoldSPINK, I962), (RowE et Gol,DSPINK, 1969). Ces données sur l'évolution du diamètre des fibres, pendant la croissance musculaire, ont été obtenues avec des techniques très diverses, dont l'influence sur les dimensions des fibres a été souvent mal étudiée. En effet, la fixation de la masse musculaire et surtout son inclusion dans la paraffine, pendant laquelle l'action de la chaleur provoque une rétraction des fibres de collagène entourant les fibres musculaires, du tiers au quart de leur longueur initiale, entraînent un rétrécissement du muscle. Ces effets ajoutés aux modifications de tonus, au cours du prélèvement du muscle, ont une action difficilement contrôlable sur la taille des fibres ; par suite il est malaisé d'obtenir des données précises. C'est pourquoi on a mis au point une technique permettant de mesurer sur coupes histologiques, des aires de sections transversales des fibres aussi voisines que possible des dimensions réelles chez l'animal vivant.

Les données bibliographiques relatives à la croissance en longueur des fibres sont, par contre, fort rares (MEARA, I947).

\section{MATÉRIEL ET MÉTHODES}

Le matériel animal provient d'une souche de lapin commun élevée au laboratoire. Les deux muscles étudiés sont le soleus, muscle rouge de la jambe, et l'accessorius latissimi dorsi, muscle mixte du bras. Ils ont été prélevés pour les deux sexes aux âges suivants : naissance, 15, 30, 70, I 50 et 360 jours, avec 5 animaux par âge et par sexe.

Les mesures d'aire de section et de longueur des fibres effectuées sur les muscles contralatéraux ont été pratiquées de la manière suivante.

\section{Mesure des aires de section des fibres}

Immédiatement après l'abattage, les muscles étudiés sont dégagés et, pour une position standard des membres sur lesquels ils sont fixés, congelés en place à une température de $-120^{\circ} \mathrm{C}$ dans le méthyle-r-butane refroidi par l'azote liquide. Après prélèvement des muscles congelés les coupes sont faites au microtome cryostat et des surfaces de faisceaux de fibres congelées sont 
mesurées en chambre froide, pour éviter leur réchauffement, à un grossissement de I zo. Ces mêmes surfaces sont par la suite mesurées au même grossissement sur les mêmes coupes, qui ont été, entretemps, fixées pendant deux minutes dans un mélange, d'éthanol absolu, d'acide acétique et de chloroforme $(v: v: v:)$, puis colorées à l'hématoxyline dioxane. Connaissant les surfaces des faisceaux de fibres et le nombre de fibres dans ces faisceaux, les aires moyennes de section ont pu être calculées d'une part pour la fibre congelée seulement et d'autre part pour la fibre congelée, puis fixée et colorée.

\section{TABLEAU I}

Comparaison de l'aire moyenne de section transversale entre fibres congelées et fibres congelées et fixées

Valeurs moyennes, $t$ écart-type $\left(\mu \mathrm{m}^{2}\right)$ (Nombre d'observations)

\begin{tabular}{|c|c|c|c|c|}
\hline \multirow[b]{2}{*}{ Animal } & \multicolumn{2}{|c|}{ Accessorius latissimi dorsi } & \multicolumn{2}{|c|}{ Soleus } \\
\hline & $\begin{array}{l}\text { Aire section } \\
\text { Moyenne } \\
\text { Fibre congelée }\end{array}$ & $\begin{array}{l}\text { Aire section } \\
\text { Moyenne } \\
\text { Fibre fixée }\end{array}$ & $\begin{array}{l}\text { Aire section } \\
\text { Moyenne } \\
\text { Fibre congelée }\end{array}$ & $\begin{array}{l}\text { Aire section } \\
\text { Moyenne } \\
\text { Fibre fixée }\end{array}$ \\
\hline 1 & $\begin{array}{c}3918,75 \pm \\
330,04 \\
(n=10)\end{array}$ & $\begin{array}{c}3922,36 \pm \\
317,53 \\
(n=10)\end{array}$ & $\begin{array}{c}3297,81 \pm \\
244,37 \\
(n=10)\end{array}$ & $\begin{array}{c}3364,20 \pm \\
268,77 \\
(n=10)\end{array}$ \\
\hline 2 & $\begin{array}{c}2710,71 \pm \\
273,63 \\
(n=10)\end{array}$ & $\begin{array}{c}2720,53 \pm \\
295,85 \\
(n=10)\end{array}$ & $\begin{array}{c}2807,33 \pm \\
167,33 \\
(n=10)\end{array}$ & $\begin{array}{c}2792,89 \pm \\
155,95 \\
(n=10)\end{array}$ \\
\hline 3 & $\begin{array}{c}2846,80 \pm \\
290,13 \\
(n=10)\end{array}$ & $\begin{array}{c}2802,07 \pm \\
317,48 \\
(n=10)\end{array}$ & $\begin{array}{c}3246,50= \\
172,25 \\
(n=10)\end{array}$ & $\begin{array}{c}3203,78 \pm \\
155,95 \\
(n=10)\end{array}$ \\
\hline 4 & $\begin{array}{c}3153,84 \pm \\
325,77 \\
(n=10)\end{array}$ & $\begin{array}{c}3167,63 \pm \\
307,56 \\
(n=10)\end{array}$ & $\begin{array}{c}3150,53 \pm \\
418,54 \\
(n=10)\end{array}$ & $\begin{array}{c}3123,55 \pm \\
338,52 \\
(n=10)\end{array}$ \\
\hline
\end{tabular}

Les résultats obtenus pour 4 muscles soleus et accessorius latissimi dorsi sont présentés dans le tableau $\mathbf{r}$.

Il n'y a pas de différence significative entre l'aire de section moyenne obtenue à partir des faisceaux congelés et celle obtenue à partir des faisceaux congelés, puis fixés et colorés. La technique a été testée à différents âges et elle permet d'obtenir des données sur l'aire de section des fibres, correspondant au tissu congelé très rapidement, qui est dans l'état structural le plus proche de l'état vivant. Pour mesurer les surfaces des faisceaux ou des fibres, les coupes sont projetées à des grossissements de I30, 200 et 500 suivant l'âge du muscle étudié, sur papier calque homogène, le contour des images étant dessiné et découpé a fin de déterminer leurs aires par pesées.

\section{Mesure des longueurs des fibres}

La mesure de la longueur des fibres est pratiquée sur des muscles congelés en place et fixés dans une solution à Io p. Ioo de formol, salée et refroidie. Les dimensions, longueur, largeur et épaisseur, mesurées sur les muscles fixés, restent identiques à celles des muscles congelés.

Des faisceaux de 3 à 5 fibres sont séparés et leurs longueurs sont mesurées à des grossissements de 230 et I3o, ce qui permet d'obtenir la longueur des fibres, qui, pour les deux muscles étudiés, vont toutes d'une extrémité à l'autre du faisceau. Pour l'accessorius latissimi dorsi, la longueur des faisceaux ne varie pas sur toute l'étendue du muscle et les faisceaux étudiés sont 
J. NOUGUÈS

\begin{tabular}{|c|c|c|c|c|c|c|}
\hline \multirow{13}{*}{ 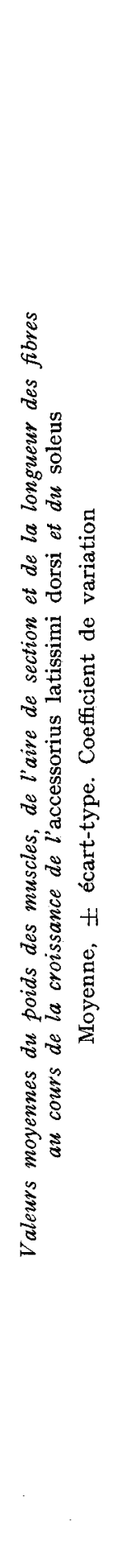 } & \multirow{6}{*}{\multicolumn{2}{|c|}{ 卷 }} & $\stackrel{80}{8}$ & 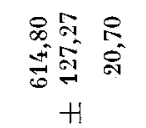 & 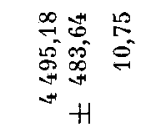 & 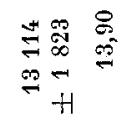 \\
\hline & & & 品 & 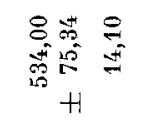 & 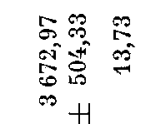 & 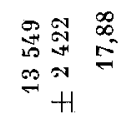 \\
\hline & & & $\therefore$ & 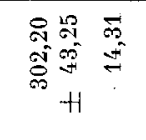 & 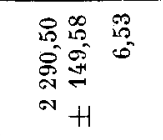 & 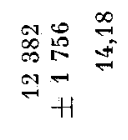 \\
\hline & & & קి & 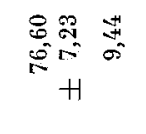 & 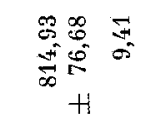 & 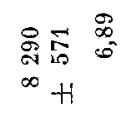 \\
\hline & & & $\stackrel{10}{\longrightarrow}$ & 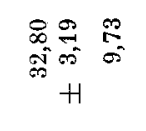 & 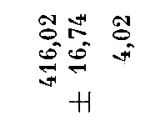 & 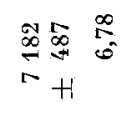 \\
\hline & & & 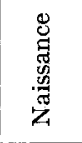 & 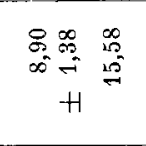 & 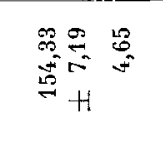 & 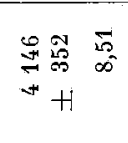 \\
\hline & 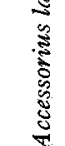 & & $\underset{8}{8}$ & 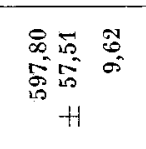 & 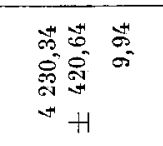 & 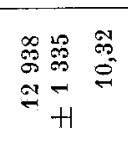 \\
\hline & & & $\stackrel{8}{\circ}$ & 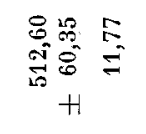 & 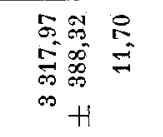 & 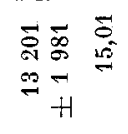 \\
\hline & & $\cong$ & $\therefore$ & 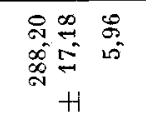 & 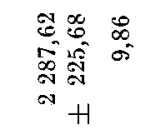 & 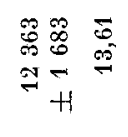 \\
\hline & & $\bar{z}$ & 间 & 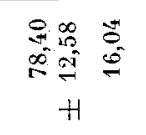 & 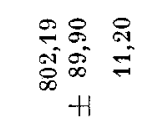 & $\begin{array}{c}\infty \\
\infty \\
\infty \\
\infty \\
\infty \\
\infty \\
\infty\end{array}$ \\
\hline & & & $\stackrel{20}{\rightarrow}$ & 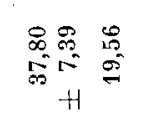 & 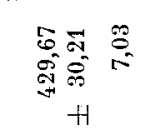 & 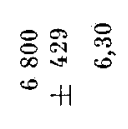 \\
\hline & & & 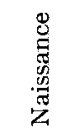 & 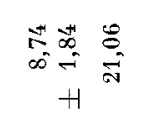 & 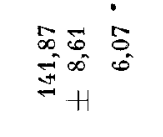 & 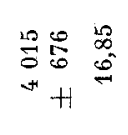 \\
\hline & $\begin{array}{l}\stackrel{0}{0} \\
\text { : } \\
\sum\end{array}$ & 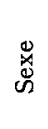 & $\stackrel{8}{4} \Xi$ & 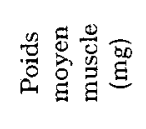 & 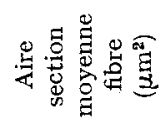 & 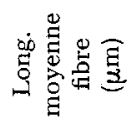 \\
\hline
\end{tabular}


HISTOLOGIE DE I,A CROISSANCE DU MUSCLE DU LAPIN

\begin{tabular}{|c|c|c|c|c|c|}
\hline \multirow{12}{*}{ కัँ } & \multirow{6}{*}{ 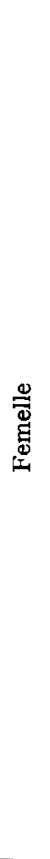 } & 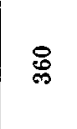 & 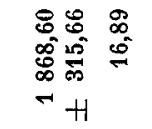 & 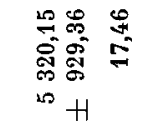 & 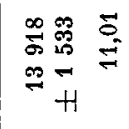 \\
\hline & & $\stackrel{9}{\stackrel{9}{2}}$ & 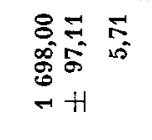 & 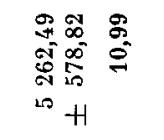 & 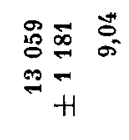 \\
\hline & & 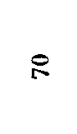 & 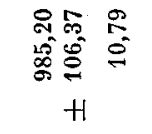 & 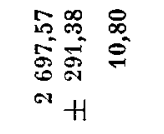 & 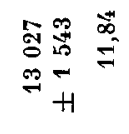 \\
\hline & & థి & 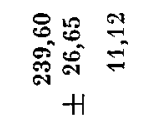 & 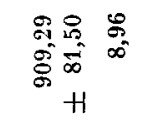 & 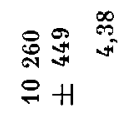 \\
\hline & & $\stackrel{12}{\rightarrow}$ & $\begin{array}{c}\mathscr{E}_{0}^{2} \infty \\
g_{\infty}^{\infty} \infty \\
+1\end{array}$ & 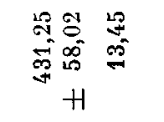 & $\begin{array}{c}0 \% \\
0 \\
0 \\
0 \\
10\end{array}$ \\
\hline & & 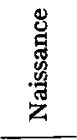 & 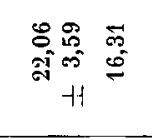 & 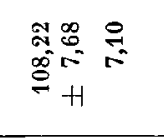 & 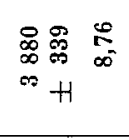 \\
\hline & \multirow{6}{*}{$\stackrel{\infty}{\sum_{i}^{\pi}}$} & 总 & 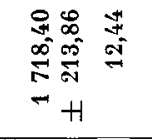 & 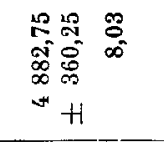 & 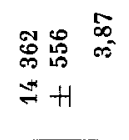 \\
\hline & & $\stackrel{8}{7}$ & 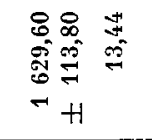 & 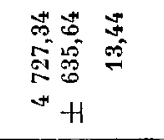 & 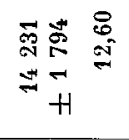 \\
\hline & & $R$ & 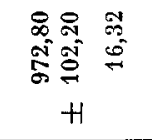 & 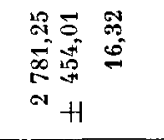 & $\begin{array}{l}\infty=\frac{\infty}{\infty} \\
\infty \\
\infty \\
\infty\end{array}$ \\
\hline & & 户ి & 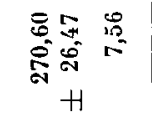 & 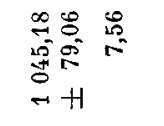 & 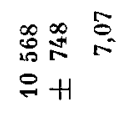 \\
\hline & & $\stackrel{10}{\longrightarrow}$ & 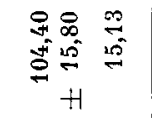 & 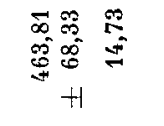 & 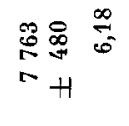 \\
\hline & & 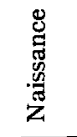 & 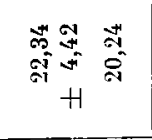 & 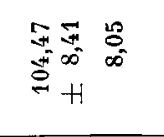 & 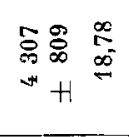 \\
\hline 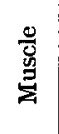 & 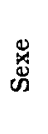 & $\frac{5}{4}=$ & 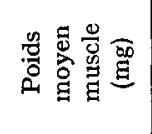 & 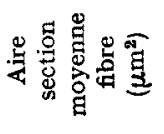 & 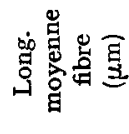 \\
\hline
\end{tabular}


prélevés dans la partie médiane. Pour le soleus, il y a une très légère différence dans la longueur des faisceaux entre les deux moitiés transversales du muscle et de ce fait les faisceaux étudiés sont toujours prélevés dans la mème moitié.

\section{Mesure des proportions de fibres musculaires et de tissu conjonctif}

L'évolution, pendant la croissance, des fractions du tissu musculaire occupé d'une part par les fibres et d'autre part par l'ensemble endomysium et périmysium a été analysée, sur les coupes qui ont servi à mesurer l'aire de section des fibres, en utilisant la méthode des points de CHALKLEY (I943). Les 289 points formés par l'intersection d'une grille à 256 carreaux ont été projetés sur des champs de coupe, pris au hasard, jusqu'à l'obtention d'un total de l'ordre de 300 points sur le conjonctif, ce qui constitue un échantillon suffisant pour avoir une variation relativement faible du pourcentage de surface occupée par les fibres et le conjonctif.

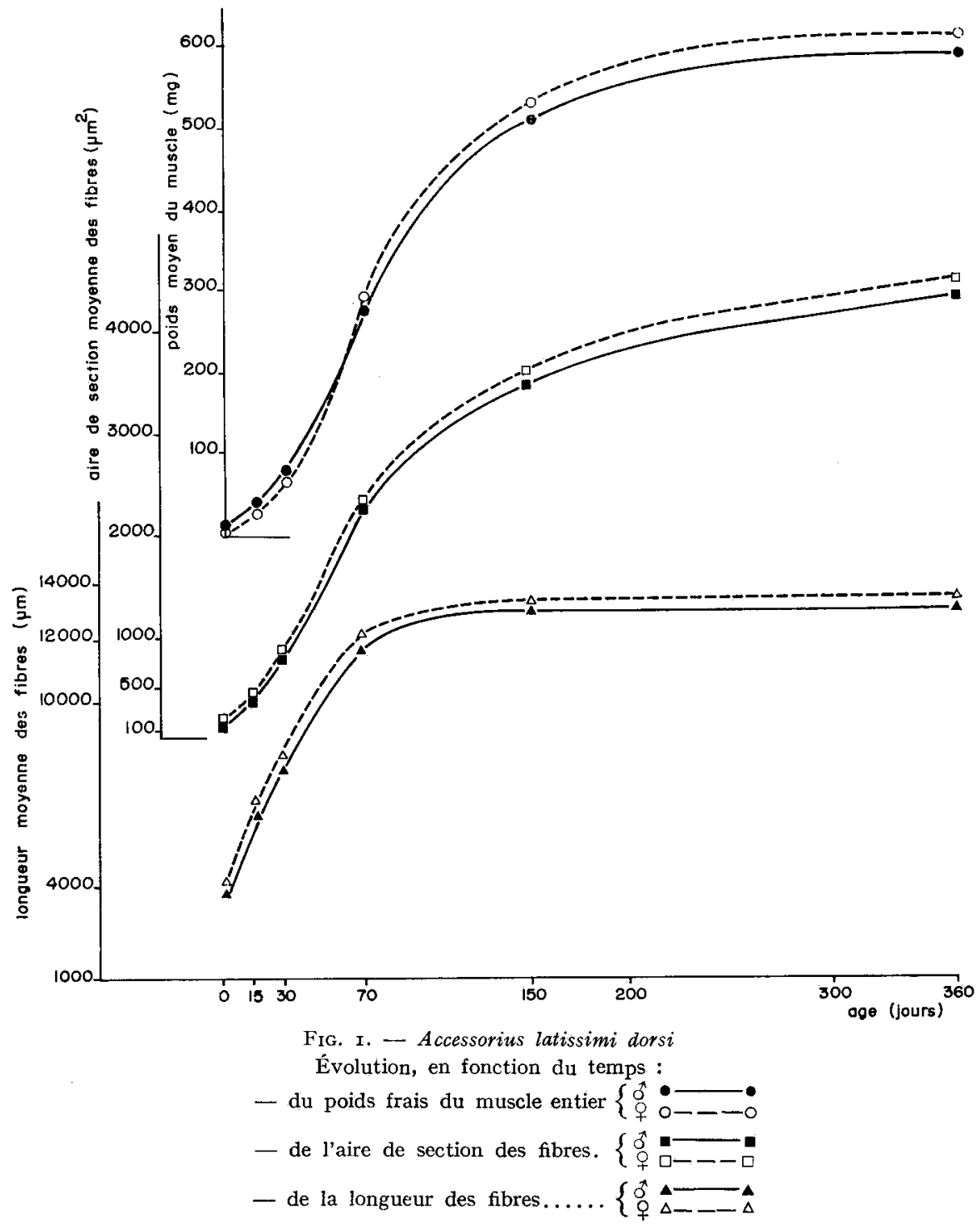




\section{RÉSULTATS}

Les valeurs moyennes des résultats concernant l'évolution du poids musculaire et l'évolution des dimensions des fibres des deux muscles étudiés sont données dans le tableau 2. Leurs représentations graphiques sont données dans les figures I et 2. Pour les deux muscles l'évolution de l'aire de section moyenne des fibres a la

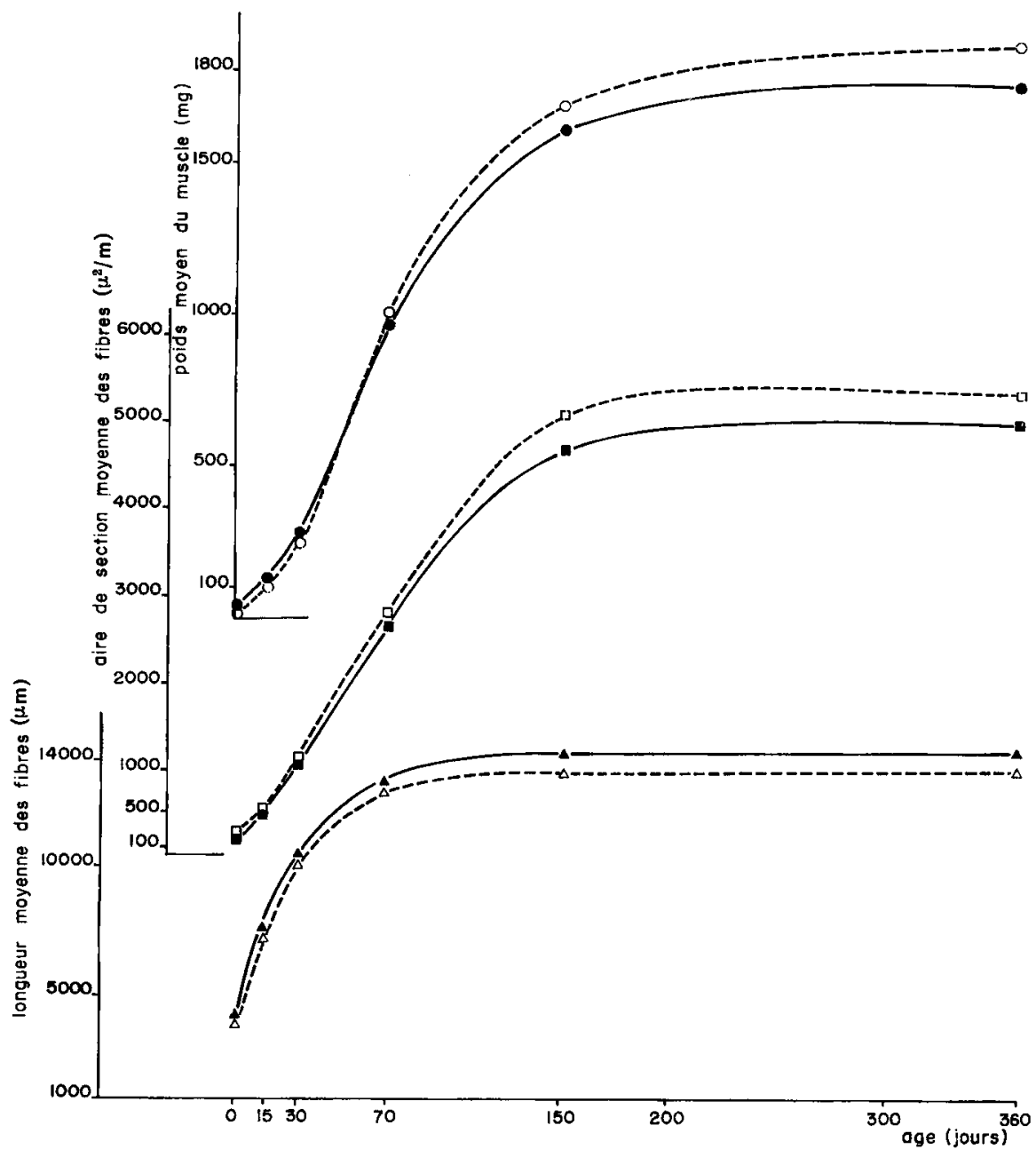

FIG. 2. - Soleus

Évolution, en fonction du temps :

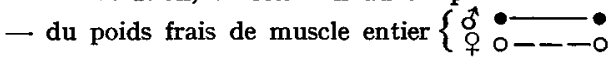

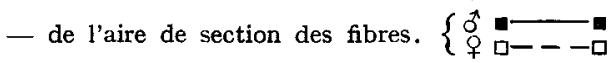

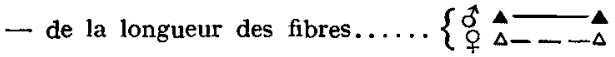


J. NOUGUÈS

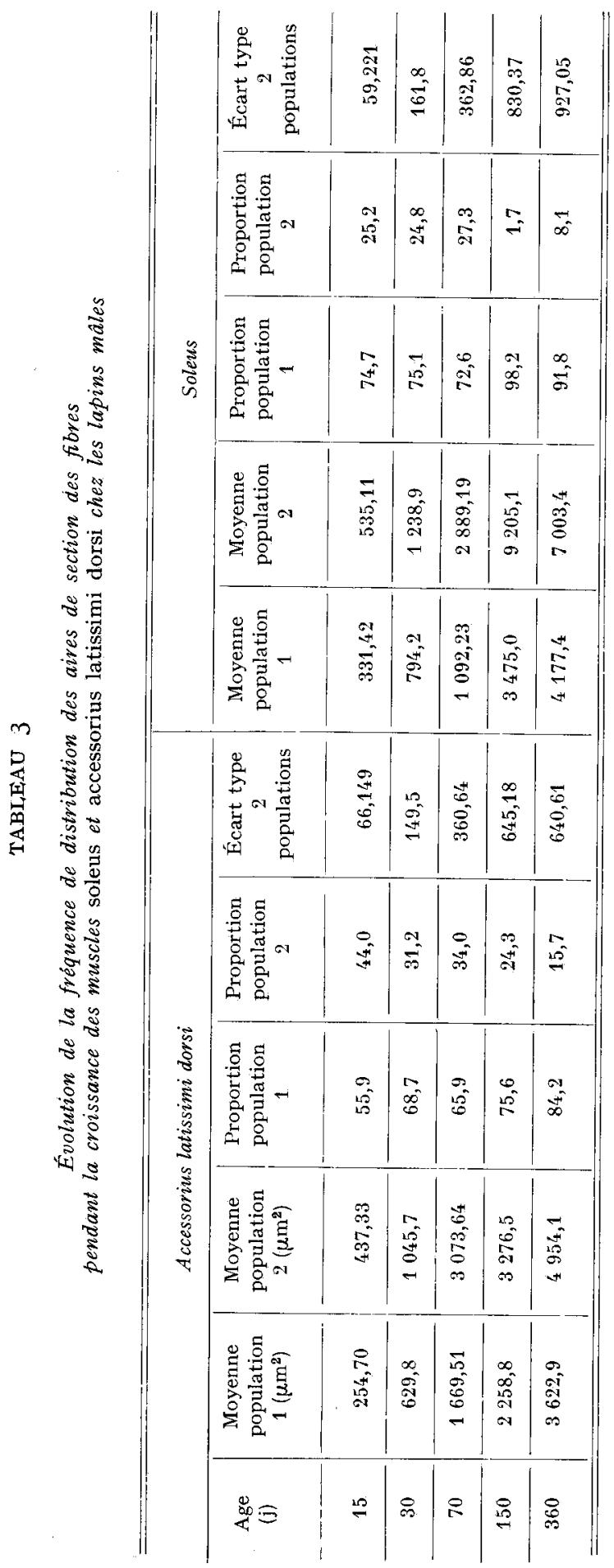


même allure que celle du poids frais des muscles. Jusque vers 60 jours, il y a un accroissement accéléré de l'aire de section des fibres, qui se ralentit par la suite. A 7o jours le poids des muscles et l'aire de section des fibres atteignent environ la moitié de leurs valeurs terminales chez les mâles et chez les femelles.

Vers I50 jours, soit pour un poids vif moyen de $3500 \mathrm{~g}$, la croissance du muscle soleus est pratiquement achevée, celle de l'accessorius latissimi dorsi se poursuit encore lentement. Ainsi, entre I5o et 360 jours, l'aire moyenne de section transversale des fibres et le poids de ce muscle augmentent respectivement de $22 \mathrm{p}$. Ioo et I5 p. IOo, contre 3 et $5 \mathrm{p}$. Ioo pour le soleus.

L'allongement des fibres est beaucoup plus précoce que leur épaississement. A la naissance les fibres atteignent déjà $30 \mathrm{p}$. Ioo de leur longueur finale alors que leurs aires de section ne font que $3 \mathrm{p}$. roo de leur valeur finale. A partir de 70 jours la croissance en longueur des fibres est pratiquement achevée et la croissance pondérale du muscle est alors, en grande partie, le résultat de la continuité de l'épaississement des fibres.

Les aires de section transversale et les longueurs des fibres ainsi que les poids frais des muscles accessorius latissimi dorsi et soleus ne sont pas significativement différents chez les lapins mâles et femelles pendant toute la période de temps étudiée, qui va de la naissance à un an.

En complément de l'étude de l'évolution de l'aire de section moyenne des fibres pendant la croissance des deux muscles, nous avons analysé l'évolution de la distribution des aires de section, chez les lapins mâles, pour observer s'il existe une ou plusieurs populations de fibres, discriminées par leurs aires de section.

La méthode des moments de RAO (I952) a été utilisée dans cette étude. L'analyse a porté sur cent observations par individu et les résultats obtenus, à chaque âge, en regroupant les individus sont présentés dans le tableau 3 .

Il existe pour l'accessorius latissimi dorsi une distribution bimodale de l'aire de section des fibres, qui est stable jusqu'à 1'âge de 360 jours. Par contre, pour le soleus, il y a passage d'une distribution bimodale à une distribution unimodale à partir de 1'âge de 70 jours. Pour le muscle accessorius latissimi dorsi il existe entre les individus, à chacun des divers âges étudiés, une convergence remarquable des analyses qui se retrouve aussi pour le muscle soleus mais uniquement jusqu'à l'âge de 70 jours. En effet, pour ce dernier à partir de $5_{50}$ jours, les résultats deviennent beaucoup plus variables entre individus du même âge, certains pouvant encore présenter à 360 jours des muscles avec une distribution bimodale des aires de section des fibres, mais avec une diminution très nette du pourcentage de la population des fibres de grande aire de section.

Le troisième paramètre de la croissance postnatale du muscle, envisagé dans cette étude, est l'évolution de l'espace du tissu musculaire occupé par le conjonctif. Les résultats obtenus, pour le sexe mâle, par la méthode de CHAIKLEY sont présentés dans le tableau 4 .

Pendant la croissance des deux muscles étudiés, il y a une diminution de la proportion d'espace occupé par le conjonctif.

Dans une étude précédente sur l'évolution du nombre des fibres, au cours de la croissance postnatale du muscle chez le Lapin, nous avons remarqué qu'à partir de I5 jours le nombre des fibres des deux muscles étudiés n'évolue pratiquement plus et qu'il est définitivement fixé à 30 jours. 


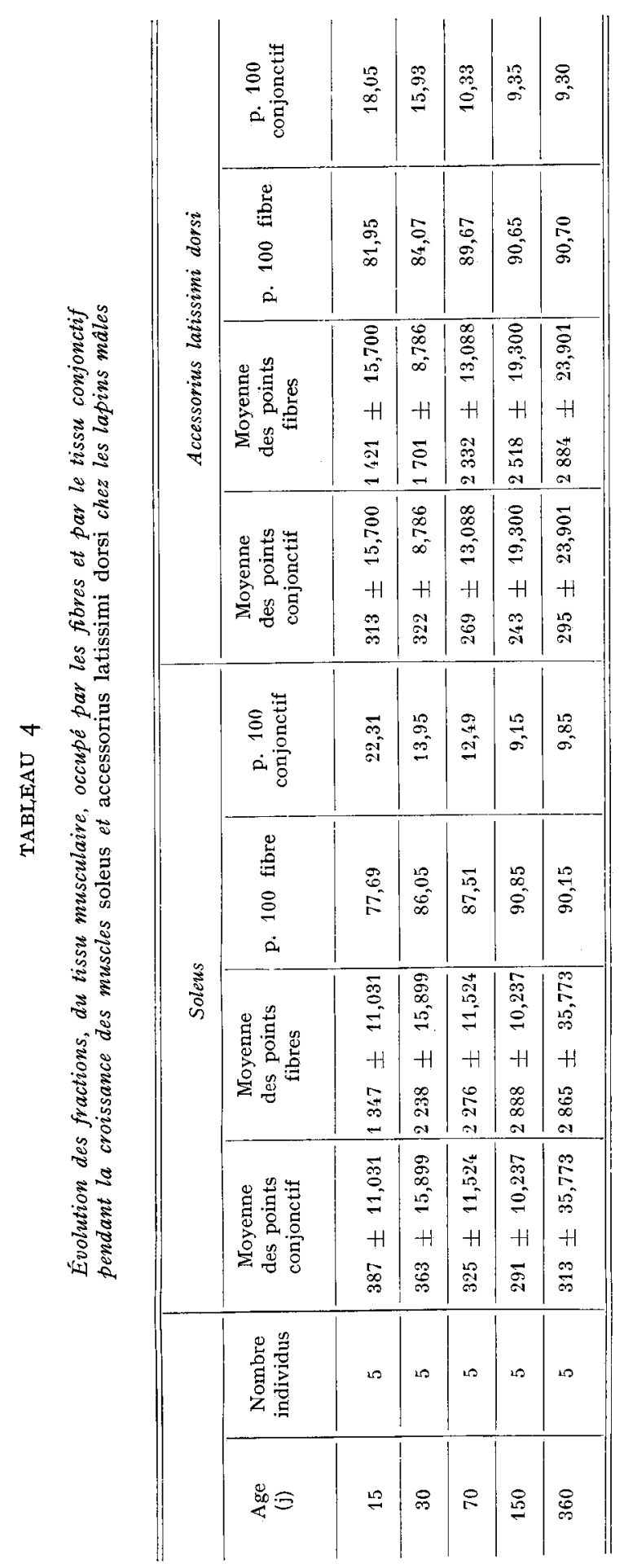


Le nombre moyen de fibres, obtenu sur I7 individus mâles, pour l'accessorius latissimi dorsi est de 7780 fibres et, sur I 2 individus mâles, pour le soleus, de 20965 fibres. Connaissant le nombre total moyen des fibres pour chaque muscle et la part moyenne du poids du muscle propre aux fibres seules, en tenant compte de la proportion de tissu conjonctif dans le tissu musculaire, on a pu calculer le poids moyen d'une fibre et étudier son évolution pendant le développement du muscle. Dans ces calculs l'influence de l'épimysium dans le poids du muscle n'a pas été considérée. Les résultats sont présentés dans le tableau 5.

TABLEAU 5

Évolution du poids d'une fibre moyenne de l'accessorius latissimi dorsi et $d u$ soleus chez les lapins males

\begin{tabular}{|c|c|c|c|c|c|}
\hline Muscle & $\begin{array}{c}\text { Nombre } \\
\text { moyen } \\
\text { fibres }\end{array}$ & $\begin{array}{c}\text { Age } \\
(\mathrm{j})\end{array}$ & $\begin{array}{c}\text { Poids moyen } \\
\text { du muscle (mg) }\end{array}$ & $\begin{array}{l}\text { Poids moyen } \\
\text { du muscle } \\
\text { propre } \\
\text { aux fibres } \\
\text { (mg) }\end{array}$ & $\begin{array}{l}\text { Poids moven } \\
\text { d'une fibre }(\mu g)\end{array}$ \\
\hline \multirow{5}{*}{ 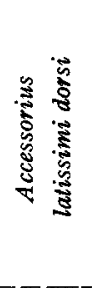 } & \multirow{5}{*}{7780} & 15 & 37,80 & 30,99 & 3,98 \\
\hline & & 30 & 78,40 & 65,85 & 8,46 \\
\hline & & 70 & 288,20 & 259,38 & 33,34 \\
\hline & & 150 & 512,60 & 466,46 & 59,95 \\
\hline & & 360 & 597,80 & 543,99 & 69,92 \\
\hline \multirow{5}{*}{ 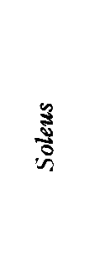 } & \multirow{5}{*}{20965} & 15 & 104,40 & 81,43 & 3,88 \\
\hline & & 30 & 270,60 & 232,71 & 11,09 \\
\hline & & 70 & 972,80 & 846,33 & 40,36 \\
\hline & & 150 & 1629,60 & 1482,93 & 70,73 \\
\hline & & 360 & 1718,40 & 1546,56 & 73,76 \\
\hline
\end{tabular}

Il apparaît que les fibres des deux muscles ont à peu près le même poids à I5 jours ; ENESCO et PUDDY (I964) ont constaté la même chose pour quatre muscles différents chez le jeune rat mâle à l'allaitement, le poids moyen des fibres étant de l'ordre de 2,I à $2,7 \mu \mathrm{g}$. Le poids moyen des fibres des deux muscles étudiés augmente considérablement avec 1'âge ; à 70 jours les fibres ont atteint environ la moitié de leur poids final. La fibre moyenne de l'accessorius latissimi dorsi a une croissance un peu plus lente que celle du soleus; ainsi à I 50 jours la fibre moyenne du soleus a atteint 96 p. Ioo de son poids final contre 86 p. Ioo pour l'accessorius latissimi dorsi. 


\section{DISCUSSION}

La croissance postnatale du muscle est surtout le résultat d'une hypertrophie considérable des fibres qui constituent de 75 à $90 \mathrm{p}$. Ioo du poids du tissu chez le Lapin.

L'allongement des fibres est beaucoup plus précoce que leur accroissement en épaisseur et il s'arrête beaucoup plus tôt. Les résultats des études effectuées au laboratoire, sur la croissance du squelette chez le lapin domestique, ont montré qu'à 70 jours le tibia, sur lequel est fixé le soleus, et l'humérus sur lequel est fixé $1^{\prime}$ accessorius latissimi dorsi, ont atteint respectivement $90 \mathrm{p}$. Ioo et $85 \mathrm{p}$. Ioo de leur longueur finale. La croissance en longueur des fibres des deux muscles étudiés s'arrête pratiquement en même temps que celle des os sur lesquels ils sont fixés.

Nos observations sont en accord avec celles de Meara (I947) obtenues chez le lapin mâle. Cet auteur a, en effet, observé un arrêt de l'allongement des fibres du gastrocnemius vers l'âge de 52 jours, c'est-à-dire à un âge beaucoup plus bas que pour l'arrêt de l'accroissement du diamètre des fibres. Cependant, ces données ne concernent que des muscles à faisceaux courts ayant des fibres de leur longueur. Ainsi MEARA a observé, dans l'étude de la croissance du muscle psoas du Lapin, dont les fibres sont plus courtes que les faisceaux, que l'allongement des fibres continuait plus tardivement et même tout au long de la croissance du muscle.

Les deux muscles étudiés ont des poids très différents alors que les aires de section transversales ou les poids moyens de leur fibres sont peu différents, ce qui montre que c'est le nombre de fibres qui est le facteur le plus important dans la détermination de la masse du muscle.

Peu d'auteurs ont étudié la forme de la distribution des diamètres des fibres et son évolution au cours de la croissance du muscle. Parmi eux RowE et GolDSPINK (I969) ont observé, graphiquement, qu'il y avait pour les muscles extensor digitorum longus et soleus de la Souris une distribution unimodale du diamètre des fibres tout au long de la croissance avec la classe de plus grande fréquence correspondant à un diamètre de $25 \mu \mathrm{m}$ à 24 semaines. Par contre, pour les muscles biceps brachii et tibialis anterior il y a apparition d'une distribution bimodale, par hypertrophie d'une certaine proportion de fibres qui passent d'un diamètre de $25 \mu \mathrm{m}$ à $40 \mu \mathrm{m}$ à l'âge de 24 semaines. D'autre part, GoLDSPINK (I962) a observé que la distribution bimodale des fibres dans le muscle biceps brachii de la Souris pouvait être modifiée selon les conditions physiologiques. Ainsi, après une période d'exercice physique intensif, il y a une augmentation de la population des fibres de grand diamètre, tandis qu'après une période de jeûne sévère, il $\mathrm{y}$ a une augmentation de la population des fibres de petit diamètre, sans qu'il y ait dans les deux cas, une modification des classes de plus grande fréquence.

Les résultats que nous avons obtenus, par une autre méthode, pour le muscle soleus du Lapin mâle sont différents de ceux obtenus par RowE et GoLDSPINK (I969) pour le soleus de la Souris. Nous observons pour le muscle soleus du Lapin, une distribution bimodale des aires de section des fibres jusqu'à l'âge de 70 jours avec les trois quarts des fibres se distribuant dans la population d'aire moyenne de section transversale la plus faible, cette distribution devenant unimodale à partir de 150 jours. 
Ces résultats seraient en accord avec ceux de NysTRöm (I968) relatifs à l'étude histochimique du développement du muscle soleus du Chat. Cet auteur a observé, entre la naissance et 20 semaines, l'apparition de deux populations de fibres discernables par leurs caractères histoenzymatiques ; ces deux populations disparaissent dans le soleus du Chat adulte, pour faire place à une seule population de fibres toutes identiques histoenzymatiquement, avec cependant la présence d'un très faible pourcentage (voisin de I) de fibres dont les caractères enzymatiques sont particuliers. Cependant l'auteur ne donne aucun renseignement sur les relations entre le diamètre des fibres et leurs types métaboliques déterminés par histoenzymologie.

En ce qui concerne l'évolution des proportions du tissu musculaire occupé par les fibres et le conjonctif, les résultats obtenus sont en accord avec ceux de la bibliographie. Ainsi, Chiakulas let Pauly (rg65) et Finesco et Puddy (I964) ont observé que la proportion de tissu conjonctif diminuait pendant la croissance du muscle chez le Rat. Ces derniers auteurs ont observé que chez les rats, en fin de croissance, le pourcentage du poids du tissu conjonctif dans le poids du muscle variait entre $\mathrm{I} 8$ et I $_{5} \mathrm{p}$. Ioo.

Reçu pour publication en juillet 1972.

\section{REMERCIEMENTS}

Les calculs concernant l'étude de l'évolution de la fréquence de distribution des aires de section des fibres ont été effectués sur l'I. B. M. I I zo de la station de Biométrie du C. N. R. F. Nous remercions M. MrLlier et ses collaborateurs pour les conseils et l'aide qu'ils ont bien voulu nous apporter.

\section{SUMMMARY}

HISTOLOGICAL STUDY OF THE POST-NATAL GROWTH

OF THE SOLEUS AND ACCESSORIUS LATISSIMI DORSI MUSCLES

IN THE COMMON RABBIT

Post-natal growth of the accessorius latissimi dorsi and soleus muscles has been studied in male and female rabbits between birth and one year.

Evolution of the following characteristics has been determined :

- Dimension of muscle fiber transversal cross-section area and lenght,

- proportion of connective tissue in the muscle tissue.

It appears that :

- The evolution of muscle fiber cross-section area resembles the ponderal evolution of muscles in terms of time. There is an accelerated period of growth of the cross-section area up to about 60 days, followed by a period of deceleration which levels off at about I5o days for the soleus and 250 days for the accessorius latissimi dorsi.

The average surface area of the transversal cross-sections and the length of fibers, as well as muscle live-weight, are not significantly different in male and female rabbits during growth of the muscles studied.

Muscle fibers grow in length much earlier than in thickness, and growth stops sooner. Growth in length is practically completed at 70 days in both muscles studied.

- The proportion of connective tissue, reprensented by the whole perimysium and endomysium, decreases in the muscle tissue while the muscles under study grow. 


\section{RÉFÉRENCES BIBLIOGRAPHIQUES}

CHALKLEY H. W., 1943. Method for the quantitative morphologic analysis of tissues. $J$. nat. Cancer Inst., 4, 47-54.

Chiakulas J. J., Pauly J. E., 1965. A study of postnatal growth of skeletal muscle in the rat. Anat. Rec., 152, 55-62.

Chrystall $\quad$ B. B., Zobrisky $\quad$ S. E., Bailey $\quad$ M. E., I969. Longissimus muscle growth in swine. Growth, 33, $361-370$.

Eliott T. S., Wigginton R. C., Corbin K. B., r943. The number and size of muscle fibers in the rat Soleus in relation to age, sexe and exercice. Anat. Rec., 85, 307-308.

ENEsco M., PUdDy D., I964. Increase in the number of nuclei and weight in skeletal muscle of rats of various ages. Ann. J. Anat., 114, 235-244.

Goldspink G., r962. Studies on postembryonic growth and development of skeletal muscle. Proc. Ir. Acad., 62, B, I35-I50.

Hammond J., Appleton A. B., 1932. Growth and development of mutton qualities in the sheep. Oliver and Boyd, Edinburg, $595 \mathrm{pp}$.

Joubert D. M., 1956. An analysis of factors influencing growth and development of the muscle fibre. J. agric. Sci. Camb., 47, 59-102.

Mac MeEkAN C. P., I940. Growth and development in the pig, with special reference to carcass quality characters. J. agric. Sci. Camb., 30, 276-344.

Meara P. J., I947. Meat studies., I. Postnatal growth and development of muscle, as exemplified by the gastrocnemius and psoas muscles of the rabbit. Onderstepoort. J. vet. Sci. Anim. Ind., 21, 329-466.

Nouguìs J., I972. Etude de l'évolution du nombre des fibres musculaires au cours de la croissance postnatale du muscle chez le Lapin. C. . Soc. Biol. 166 I65-I72.

Nyström B. O., r968. Histochemistry of developing cat muscle. Acta. Neurol. scand., 44, 405-439.

RAO C. R., r952. Advanced statistical methods in biometric research. Wiley, New York.

Rowe R. N. D., Goldspink G., I969. Muscle fibre growth in five different muscle in both sexes of mice. I. Normal mice. J. Anat., 104, 519-530.

Staun H., ig63. Various factors affecting number and size of muscle fibers in the pig. Acta Agric. scand., 13, 293-322. 